\title{
PPPM in neurodegenerative diseases
}

\author{
BIOMARKERS FOR PREDICTION AND TARGETED \\ PREVENTION OFALZHEIMER'S AND PARKINSON'S \\ DISEASES: EVALUATION OF DRUG CLINICAL \\ EFFICACY \\ Mandel $S A^{1}$, Korczyn $A D^{2}$ \\ ${ }^{1}$ Eve Topf Center for Neurodegenerative Diseases Research \\ and Department of Molecular Pharmacology, Faculty of \\ Medicine, Technion, Haifa, Israel \\ ${ }^{2}$ Tel-Aviv University Medical School, Ramat-Aviv, Israel
}

Neurodegenerative diseases like Parkinson's disease (PD) and Alzheimer's disease (AD) are considered disorders of multifactorial origin, inevitably progressive and having a long preclinical period. Currently, the clinical diagnosis of PD can be made when motor symptoms occur, though the disease has originated several years earlier. Furthermore, at its initial stages PD may be confounded by other diseases, such as essential tremor, multiple system atrophy (MSA) and progressive supranuclear palsy (PSP). Similar to PD, the pathological process characteristic of $\mathrm{AD}$ begins decades before the first symptoms of cognitive dysfunctions, thus making it difficult to reliably identify pathology based on the clinical phenotype alone. AD may share clinical features with many other dementing disorders including vascular dementia, dementia with Lewy bodies, PSP and others. Therefore, the availability of biological markers or biomarkers (BMs) for early disease diagnosis will impact the management of $\mathrm{AD}$ and $\mathrm{PD}$ in several dimensions; it will 1) help to capture high-risk individuals before symptoms develop, a stage where prevention efforts might be expected to have their greatest impact; 2) provide a measure of disease progression that can be evaluated objectively, while clinical measures are much less accurate; 3) help to discriminate between true AD or PD and other causes of a similar clinical syndrome; 4) delineate pathophysiological processes responsible for the disease; 5) determine the clinical efficacy of novel, disease-modifying (neuroprotective) strategies.
BMs have been defined as "cellular, biochemical or molecular alterations that are measurable in the biological media such as human tissues, cells or fluids". BMs for detection of neurodegenerative disorders can be divided into three main categories: genetic, neuroimaging, and biochemical. Normally, neuroimaging and biochemical BMs mark the presence of pathology, while genetic markers can only serve for risk assessment. Neurochemical BMs measured in the periphery by means of proteomics/ metabolomics in blood plasma, CSF and other tissues, may be useful adjuncts to imaging and clinical assessment tools for $\mathrm{AD}$ and $\mathrm{PD}$. They may also provide valuable information about pathogenic mechanisms during clinical testing of neuroprotective/disease modifying drugs, which is especially relevant to personalized treatment.

Since both AD and PD have a long preclinical period, future efforts should be focused on this time window to begin a neuroprotective treatment. Early screening, detection, and diagnosis of $\mathrm{AD}$ and $\mathrm{PD}$ will allow intervention with disease modifying therapies at earlier stages and thus may potentially improve clinical outcome. Large multicenter studies are warranted to evaluate the diagnostic value of the combined application of BMs with multiple modalities for prediction, targeted prevention and evaluation of disease modifying therapies in $\mathrm{AD}$ and $\mathrm{PD}$.

\section{ALZHEIMER'S DISEASE: DIAGNOSTICS, PROGNOSTICS AND THE ROAD TO PREVENTION} Grossman I

Cabernet Pharmaceuticals, USA

Alzheimer's disease (AD) presents one of the leading healthcare challenges of the 21 st century, with a projected world-wide prevalence of $>107$ million cases by 2025 . While biomarkers have been identified which may correlate with disease progression or sub-type for the purpose of 
disease monitoring or differential diagnosis, dependable prediction of late onset disease risk has not been available until now. This deficiency in reliable predictive biomarkers, coupled with the devastating nature of the disease, places $\mathrm{AD}$ at a high priority for focus by predictive, preventive and personalized medicine (PPPM). Recent data, discovered using phylogenetic analysis, suggest that a variable length poly-T sequence polymorphism in the TOMM40 gene, adjacent to the $A P O E$ gene, is predictive of risk of $\mathrm{AD}$ age-of-onset. This finding has been confirmed in several independent cohorts, thus offering hope for reliable assignment of disease risk within a 5-7 year window. This scientific finding is now being utilized in a pharmacogenetic-enrichment global clinical trial for the first $\mathrm{AD}$ preventative therapy. This clinical trial will also prospectively validate the utility of this marker as a standalone prognostic of incipient disease.

\section{DISCOVERY, DEVELOPMENT, VALIDATION AND QUALIFICATION OF MARKERS WITH CLINICAL UTILITY TO THE DELAY OF ONSET OF ALZHEIMER'S DISEASE CLINICAL TRIAL}

Roses $A D$

Duke University and Zinfandel Pharmaceuticals, Inc., USA

A novel clinical trial design is being used for the simultaneous development of a predictive test algorithm, a companion genetic diagnostic [predictor] and testing the efficacy of a drug to delay the onset of Alzheimer's disease (AD).

The TOMM40 gene encodes the translocase of the outer mitochondrial membrane pore subunit, and is in linkage disequilibrium with the APOE gene. The length of a poly-T variant ("523") within intron 6 of TOMM40 has been shown to predict the age of onset of late onset Alzheimer's disease (LOAD). These data allow the design of a prospective epidemiologic-ascertained longitudinal study to assess the onset of neuropsychological signs of cognitive impairment and the progression of clinical impairment. By using the group predictions to segment subjects' risk based on their age, APOE genotype, and TOMM40-523 genotype, the positive and negative predictive values obtained from placebo treated subjects can be computed at the end of the study. These prospective data are necessary before the data can be used as a prognostic test for the risk of disease and its age of onset.

The design of the clinical study to assess the clinical utility of a predictive algorithm provides the opportunity to simultaneously perform a clinical trial. This trial will assess whether a drug can delay onset of cognitive impairment of the type leading to AD. The design of OPAL [Opportunity to Prevent Alzheimer's disease] was considered with the
FDA Voluntary Exploratory Data Submission group before any drug was nominated and was determined to be a welldesigned pharmacogenetic-assisted clinical trial.

It is important to point out that the predictions proposed for the study are simply being used to segment high and lower risk groups for the purpose of evaluation of the predictive value [both positive and negative] and the effect of a safe drug for which there is a rationale to slow or prevent the AD process. Clinical utility demands that the putative predictive algorithms be tested before application in normal individuals for so-called AD predictions. The study design provides the opportunity to demonstrate that a drug delays onset, so that the use of the clinical predictive data is more than just a prediction, but an opportunity for preventive therapy.

In January 2011, a partnership was established by Zinfandel Pharmaceuticals, Inc. and Takeda Pharmaceuticals, Inc. to develop pioglitazone, a drug approved for treatment of diabetes mellitus, to delay the onset of $\mathrm{AD}$. An up-to-date report of the status of the trial, particularly concerning the procedures for registering a companion diagnostic [predictive] with a therapeutic agent, will be presented. The first steps are: 1- engagement with the regulatory authorities based on newly published regulatory guidelines; 2- identification of normal subject groups; and 3- organization of the resources for periodic neuropsychology testing of hundreds of people at each study site for an extended period of time.

NEW ANIMAL MODELS OF PROGRESSIVE NEURODEGENERATION: TOOLS FOR IDENTIFYING PRESYMPTOMATIC THERAPEUTIC TARGETS Adams-Marriott $A L^{1}$, Shaw $C A^{2}$, Tasker $R A^{1}$

${ }^{1}$ Department of Biomedical Sciences, University of Prince Edward Island, Charlottetown, PEI, Canada

${ }^{2}$ Department of Opthalmology, University of British Columbia, Vancouver, BC, Canada

Mental and neurological disorders are increasingly prevalent and constitute a major societal and economic burden worldwide. The impact of these disorders has traditionally been underestimated by epidemiological data that have relied on mortality rather than morbidity. According to the World Health Organization mental and neurological disorders are responsible for only about $1 \%$ of deaths but account for almost $11 \%$ of the global disease burden [http:// www.who.int/mental health/neurology/en]. Further, due to increased life expectancy and the ageing of general populations in both developed and developing countries this number is expected to rise to $14.7 \%$ by 2020 . Many of the most socially and economically devastating neurological diseases and disorders are characterized by progressive 
neurodegeneration. Symptoms of many forms of progressive disease often appear in late adolescence or early adulthood and then become increasingly severe with increasing age. The neurodegenerative process however, usually begins long before the onset of clinically diagnosed symptoms. Thus, in many cases a precipitating event occurring much earlier in life, often around the time of birth or in early childhood, appears to initiate a degenerative cascade that proceeds undetected for some time prior to the onset of clinical signs. Presymptomatic detection of disease therefore represents a largely unexplored opportunity for therapeutic intervention. This general concept is depicted in Figure.

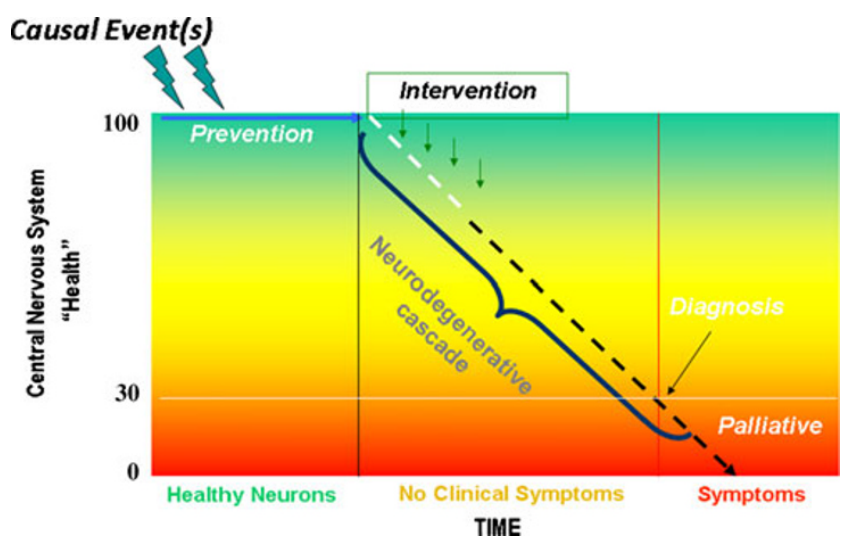

Understanding the aetiology, and initiation, of disease often relies on animal models, as does the development of new therapeutic strategies. But while there are a multitude of animal models that mimic specific features of end-stage disease for almost all neurological conditions, most of these models have been created to identify new therapeutants to alleviate symptoms, and hence do not allow study of the development of disease. Understanding disease development is the key to identifying early intervention strategies consistent with the concept in Figure. There exists, therefore, a largely unmet need to develop and exploit animal models designed to understand the origins and progression of neurological disease. This presentation will introduce several examples of recently developed and characterized animal models of progressive neurodegenerative disease. Further, it will explore the concept of models that can be used to study co-morbidities between end-stage disease states. Developing new predictive and preventative therapeutic strategies, as well as identifying novel and reliable biomarkers and drug targets for presymptomatic intervention requires a paradigm-shift, whereby the goal is to predict and treat generalized neurologic dysfunction in the early stages rather than focusing drug development on late-stage disease-defined symptoms and palliative care.

\section{RISK ASSESSMENT IN GLAUCOMA DEVELOPMENT}

Golubnitschaja $\mathrm{O}^{1,2}$, Yeghiazaryan $\mathrm{K}^{1,2}$, Flammer $J^{2,3}$

${ }^{1}$ Department of Radiology, Rheinische Friedrich-WilhelmsUniversity of Bonn, Germany

${ }^{2}$ European Association for Predictive, Preventive \& Personalised Medicine, www.epmanet.eu

${ }^{3}$ Department of Ophthalmology, University Hospital Basel, Switzerland

Particularities of the neurodegenerative disease Glaucoma

Neurodegenerative eye disease glaucoma is the second leading cause of blindness with estimated 67 million patients worldwide. Molecular pathomechanisms of glaucoma demonstrate both a considerable overlap with and remarkable particularities compared to other neurodegenerative disorders e.g. Alzheimer's disease. Identification of pathology-specific molecular-sets is essential to develop advanced diagnostic approaches.

Glaucomatous optic neuropathy (GON) is characterised by a combination of a loss of retinal ganglion cells and their axons with disease typical optic nerve head (ONH) atrophy. The aetiology of glaucoma and risk factors are only partially known (Fig. 1).

\section{KNOWN RISK FACTORS:}
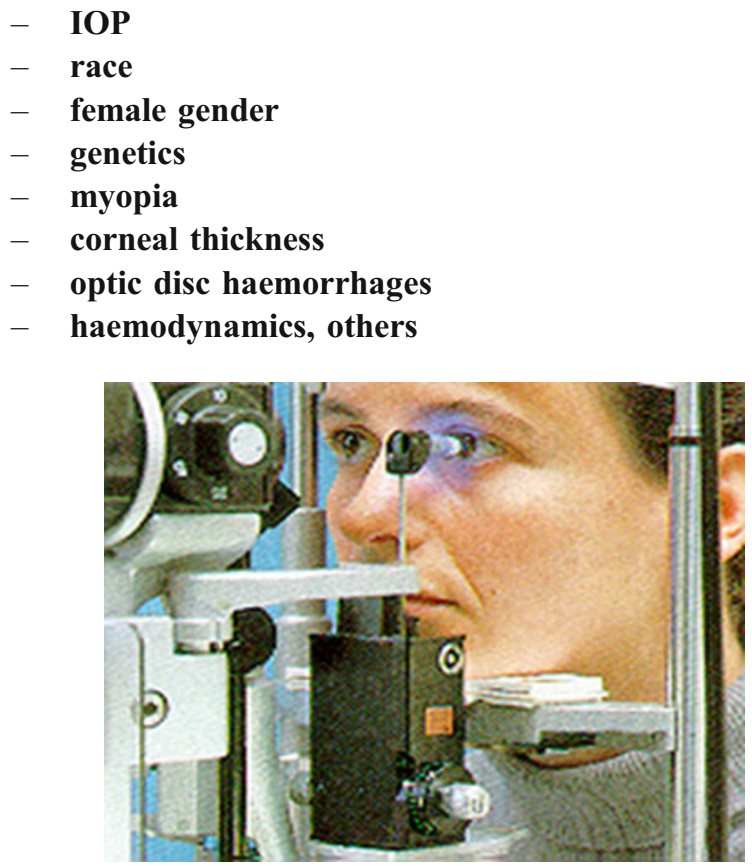

Fig. 1. Risk factors in glaucoma and measurement of intra-ocular pressure (IOP). 
IOP (intra-ocular pressure) is considered the most important and only modifiable risk factor in glaucoma. Although elevated IOP has been shown to be the major risk factor, there is a cohort of patients, sometimes even at younger ages with normal IOP developing normal-tension glaucoma (NTG).

Impact of vascular component and relevance of gene expression patterns in blood for glaucoma pathology

A wealth of literature points to the potential importance of haemodynamics in glaucoma pathology, but randomized controlled trials are not yet available. Healthy individuals with Vasospastic Syndrome demonstrate molecular profiles in blood similar to those of glaucoma patients [1]. Particular predisposition of vasospastic individuals to glaucoma is discussed in the recent review [2].

Key molecular pathways affected by glaucoma pathology: Sub-cellular imaging and expression patterns in blood as the reliable platform for early/predictive diagnosis
Key molecular pathways affected by glaucoma pathology have been recently summarized in the book specifically dedicated to predictive diagnostics and personalized treatment [3].

The majority of molecules altered in glaucoma belongs to well-defined functional gene-groups which, altogether, represent molecular pathways affected by the pathology: stress response, apoptosis and DNA-repair, adhesion, blood-brain-barrier-breakdown, tissue remodeling, transcription regulation, multi-drug resistance and energy metabolism.

\section{Outlook}

Currently a clinical application of the test is under consideration, and a nanotechnology which should provide a possibly easy and cheap routine application of the test is under development. Possible types of molecular alterations that can be considered for predictive glaucoma diagnosis and corresponding detection technologies are summarized in Table 1.

Table 1. Types of glaucoma-specific molecular alterations that can be potentially used for development of advanced tools for early and predictive diagnosis. Data taken from [4].

\begin{tabular}{|c|c|c|}
\hline Type of molecule & Possible type of alteration & Detection technology \\
\hline \multirow{4}{*}{$\begin{array}{l}\text { Chromosomal DNA, } \\
\text { mitochondrial DNA }\end{array}$} & 1. (Oxidative) damage* & - $\underline{\text { Comet assay }}$ \\
\hline & 2. Mutations* & - Disease genomics \\
\hline & 3. Polymorphism* & - $\underline{\text { PCR }}, \underline{\text { Restriction analysis, etc. }}$ \\
\hline & 4. Methylation status of $\mathrm{CpG}$ islands** & - Methylation-specific PCR \\
\hline \multirow[t]{3}{*}{ mRNA } & 1. Multiple alterations in expression patterns* & - Disease transcriptomics: \\
\hline & & $\begin{array}{l}\text { Subtractive hybridization, Expression array, } \\
\text { Reverse-Transcriptase-PCR, Real-Time-PCR, etc. }\end{array}$ \\
\hline & 2. Reduced mRNA editing & - Reverse-Transcriptase-PCR \\
\hline \multirow[t]{5}{*}{ Proteins } & 1. Multiple alterations in expression patterns* & - Disease Proteomics: 2D-PAGE, MALDI-TOF, \\
\hline & 2. Posttranslational modification* & - Western-blot, Activity tests \\
\hline & & (e.g. Zymography for gelatinases) \\
\hline & 3. Phosphorylation status* & - Activity tests \\
\hline & 4. Protein misfolding* & - Activity tests \\
\hline $\begin{array}{l}\text { Metabolites (signaling molecules, } \\
\text { amino acids, plasma hormones, etc.) }\end{array}$ & Altered profiles* & $\begin{array}{l}\text { Disease Metabolomics: Comparative blood plasma } \\
\text { metabolites profiling, HPLC, Activity tests, etc. }\end{array}$ \\
\hline
\end{tabular}

*Types of molecular alterations reported for glaucoma pathology.**Data indicating this kind of alteration collected in our laboratory, which, however, have not been published until now.

\section{References}

1. Yeghiazaryan K, Flammer J, Orgül S, Wunderlich K, Golubnitschaja O (2009). Vasospastic individuals demonstrate significant similarity to glaucoma patients as revealed by gene expression profiling in circulating leukocytes. Mol Vis, 15:2339-2348.
2. Yeghiazaryan K, Flammer J, Golubnitschaja O. Predictive molecular profiling in blood of healthy vasospastic individuals: clue to targeted prevention as personalised medicine to effective costs. EPMA J. 2010;2:263-72.

3. Golubnitschaja O, Yeghiazaryan K, Orgül S, Flammer J. What are the biomarkers for Glaucoma? In: Golubnitshaja O, editor. 
Predictive diagnostics and personalized treatment: dream or reality. New York: Nova Science; 2009. p. 375-96.

4. Golubnitschaja O, Yeghiazaryan K, Flammer J. Key molecular pathways affected by glaucoma pathology: is predictive diagnosis possible? EPMA J. 2010;2: 237-44.

\section{TECHNOLOGICAL ASPECTS OF PERSONALIZED APPROACHES IN TREATMENT OF AMYOTROPHIC} LATERAL SCLEROSIS

Benkler $C^{1}$, Offen $D^{1}$, Melamed $E^{1}$, Amit $T^{2}$, Mandel $S^{2}$, B.H. Youdim $M^{2}$, Weinreb $O^{2}$

${ }^{1}$ Felsenstein Medical Research Center, Tel Aviv University, Tel-Aviv, Israel

${ }^{2}$ Eve Topf Centers of Excellence for Neurodegenerative Diseases Research, Rappaport Family Research Institute, Technion-Faculty of Medicine, Haifa, 31096, Israel

Treatment of amyotrophic lateral sclerosis (ALS) has been fueled, in part, by frustration over the shortcomings of the symptomatic drugs available, since these do not impede the progression of the disease. Currently, over 150 different potential therapeutic agents or strategies have been tested in preclinical models of ALS. Unfortunately, therapeutic modifiers of murine ALS have failed to be successfully translated into strategies for patients, probably because of differences in pharmacokinetics of the therapeutic agents, route of delivery, inefficiency of the agents to affect the distinct pathologies of the disease or inherent limitations of the available animal models. Given the multiplicity of the pathological mechanisms implicated in ALS, new therapies should consider the simultaneous manipulation of multiple targets. Additionally, a better management of ALS therapy should include understanding the interactions between potential risk factors, biomarkers and heterogeneous clinical features of the patients, aiming to manage their adverse events or personalize the safety profile of these agents. Indeed, novel therapeutic approaches employed in preclinical models of ALS, including iron-binding brain permeable pluripotential neuroprotective compounds, gene therapy, cell replacement therapies or a combination of both can offer improved alternative therapies for ALS. Another viable therapeutic approach is transplantation of stem cells genetically engineered, or otherwise induced to secrete various neurotrophic factors, known for their role in survival, development and maintenance of motor neurons. Additionally, it has been considered that multimodal neuroprotective iron chelating compounds that possess neuritogenic effect and regulatory activity of neurotrophic factors, may mediate a regeneration process of motor nerves, and induce neuro-differentiation and sprouting of axons. These therapeutic strategies take into account the various challenging features of ALS and the interplay of genetic and environmental risk factors, to provide superior efficacy in translation from preclinical studies to the clinic.

\section{POPULATION IMAGING FOR DISEASE PREDICTION AND PREVENTION}

\section{Krestin GP}

Department of Radiology, Erasmus MC, University Medical Center Rotterdam, The Netherlands

Imaging is fundamental to basic and applied life sciences research. Life scientists use medical imaging techniques to understand living systems at both the molecular and the physiological level, from biological model systems to patients. Imaging allows non- or minimally-invasive assessment of structural and functional changes in intact organisms that may reflect specific pathology. Recent developments in image data acquisition and analysis make it feasible to use these techniques on a large scale. This makes it possible to investigate specific pathophysiological substrates of disease in a pre-symptomatic phase, in an epidemiological context, and at the population level.

It is now widely recognized that we need biomarkers to improve the accuracy of diagnoses early in the disease process and to monitor changes in organs of individuals even before the first clinical symptoms of disease manifest. Although biochemical markers have promising value in the early stages of diseases, many of them are non-specific and do not allow localization of the ongoing functional and structural alterations. Imaging biomarkers have a substantial contribution in detecting, localizing, and determining the extent of disease. Identifying imaging biomarkers and risk factors of presymptomatic disease and developing of predictive models for functional and structural alterations requires large, prospective epidemiological studies in unselected populations in which sub-clinical pathology can be assessed.

Population imaging is the large-scale application and analysis of medical images in controlled population cohorts. Population imaging aims to find imaging biomarkers that allow prediction and early diagnosis of diseases and preventive therapy. Population imaging will provide further insight into what roles risk factors play, both qualitatively and quantitatively, in the morphologic substrates underlying disease development. Dedicated infrastructures and large-scale collaborations are needed in order to extract meaningful information from population imaging studies. Correlation with outcomes and genetic information may provide further insight into risk profiles and disease prediction

A recent example is the increasing interest in cerebral microbleeds. Imaging sequences were developed to 
increase the conspicuity of hemosiderin deposits in the brain. These showed that such deposits, the so called 'microbleeds', are present in $20 \%$ of the elderly. They are thought to reflect vessel fragility, on the basis of either hypertensive vascular damage or amyloid angiopathy. A hypothesis that remains to be investigated is that persons with microbleeds are at an increased risk of symptomatic intracerebral hemorrhage. If true, the riskbenefit ratio of anticoagulant therapy to prevent ischemic brain events would differ between persons with and without co-existing microbleeds.

Population imaging has many potential areas of application for disease prediction and prevention. Examples include intima-media thickness of carotid artery for arteriosclerosis detection, coronary calcium scoring for coronary artery disease, size of the hypocampus in predicting neurodegeneration, etc. Imaging may become the key to identifying people that could benefit from preventive intervention for many more diseases. Additionally, structural and functional organ changes that reflect (incipient) clinical disease can be used as outcome measures in a variety of studies, including clinical trials and etiologic research as well as diagnostic and prognostic studies.

THE ROLE OF OPTICAL COHERENCE TOMOGRAPHY IN PREDICTING AND MONITORING DISEASE ACTIVITY IN MS

Dörr J, Paul F

Charité Berlin, Germany

Increasing evidence documents that neuronal and axonal damage within the central nervous system (CNS) contributes substantially to the development of permanent disability in multiple sclerosis (MS). However, in vivo quantification and longitudinal monitoring of neurodegenerative processes remain a challenging task. Whole brain atrophy expressed by brain parenchymal fraction (BPF) is a frequently used MRI-based surrogate parameter for neurodegeneration within the CNS and an inverse relation of BPF and disability progression has been consistently demonstrated. However, all MRI-based measures of brain atrophy have some important disadvantages. Besides limited availability, time consumption and costs, crucial confounders such as hydration status, inflammation, demyelination and age have to be accounted for. Thus, a need for reliable, inexpensive and easily assessable complementary surrogate markers for neuroaxonal degeneration still remains. During the past two decades, optical coherence tomography (OCT) has emerged into a fascinating tool for the non invasive and reproducible in vivo studying of retinal neuroaxonal damage. In MS patients, OCT has been consistently shown to detect thinning of the peripapillary retinal nerve fiber layer (RNFL) which is most probably due to a diffuse damage of retinal axons and at least in part independent of a previous optic neuritis (ON). Moreover, the determination of total macular volume (TMV) has been suggested as a marker for neuronal loss in MS patients. Therefore, OCT might be a valuable tool for quantification and monitoring of both axonal and neuronal damage in MS and thus a surrogate marker of disease activity and progression. However, data on association between retinal nerve fiber layer thickness (RNFLT) and MS-determining parameters such as disease severity and disease duration are still inconsistent. Whereas some studies found an association between RNFLT and disease duration both parameters were not related in other studies. Furthermore, data regarding the relation between OCT parameters and MRI measures for neurodegeneration are not yet consistent and a consensus on the most relevant parameter has yet to be reached. In this contribution, we will summarize and update the current data on the value of OCT in predicting and monitoring disease activity in MS and delineate the future work which remains to be done in order to improve the utility of OCT as a valuable predictive biomarker in MS and other neuroinflammatory and neurodegenerative diseases.

\section{URO- FECAL DISORDERS AND DYSPRAXIA. FMRI AS AN USEFUL TOOL FOR PERSONALIZED MEDICINE. PRELIMINARY RESULTS}

Ventimiglia B, Tsirgiotis A, Motta S, Puglisi T, Trovato G European Neurosciences Study Group, Servizio di Neuro Urologia ed Urodinamica, Dipartimento delle Specialità Medico Chirurgiche, Università di Catania, Italy

Introduction: Developmental dyspraxia is due to an impairment of the motor learning function; $4-5 \%$ of patients have also associated uro-fecal incontinence, enuresis and encopresis. This disease can affect planning of movements and co-ordination as a result of brain messages not being accurately transmitted to the body. It does not affect overall intelligence or ability, but just particular aspects of development: dyspraxia affects the planning of what to do and how to do it. It is associated with problems of perception, language and thought. It could be due to a partial or absolute deficiency of the mirror neurons development, which is responsible for the differential maturation of motor chains in the cortices. 
Learning and consolidation of the experience seems to occur through the formation of neural circuits that allow a mirror action done with as little effort as possible and lower neuronal metabolic intracerebral expenditure. Dyspraxic patients seem unable to form the mirror neuronal circuits through the common factors of experience and then, every day, it is as if they were to compare with a world known for the first time. For example, they often do not speak well because they cannot store the association between the sound of a word to the thing or person of reference.

The control of urination, the neuronal circuits that allow us to urinate as desired, is a very long corticalization process (bowel control occurs long after the child learns to talk or walk).

Functional magnetic resonance imaging can be used as an experimental paradigm for exploring mirror neurons activity and coordination during voluntary or imitative acts.

The purpose of this preliminary study was to assess if Functional Magnetic Resonance Imaging (fMRI) can predict the impairment of the process of formation of mirror neurons in Dyspraxic patients with uro-fecal incontinence with the goal of managing them earlier with a personalized approach.

Materials and methods: In 2010, we have studied six patients, four males and two females, 18-24 years old, referred for enuresis and encopresis and already diagnosed as dyspraxic. All patients were studied by routine laboratory tests and by Holter Electroencephalography with night urodynamic assessment. All subjects were examined with full and empty bladder by fMRI and multi-stage analysis with coincidence; auditory and visual evocative tests of micturition were administered. After 6 months of medical and rehabilitation treatment patients were again studied by fMRI.

Results: All the six patients showed by fMRI uncoordinated and abnormal activation of F5cortical centers, suggesting mirror neurons impairment: two patients (Group A) had detrusor overactivity, nocturnal enuresis and encopresis, and were treated by parasympathicolytic agents and behavioral physical and motor therapy, with bladder retraining. Four patients without urodynamic abnormalities but with enuresis and encopresis were treated by desmopressin and behavioral physical and motor therapy. At time 1, after treatment in Group A encopresis and enuresis disappeared and fMRI normalization of mirror response was observed. In group B, three patients achieved continence in wakefulness, encopresis decreased, but enuresis remained unchanged. One patient had no benefit. In this group B- no responders patients - the fMRI showed no difference from the baseline while in the other two patients BOLD (Blood-oxygen-level dependence) hyperactivation appeared very late.

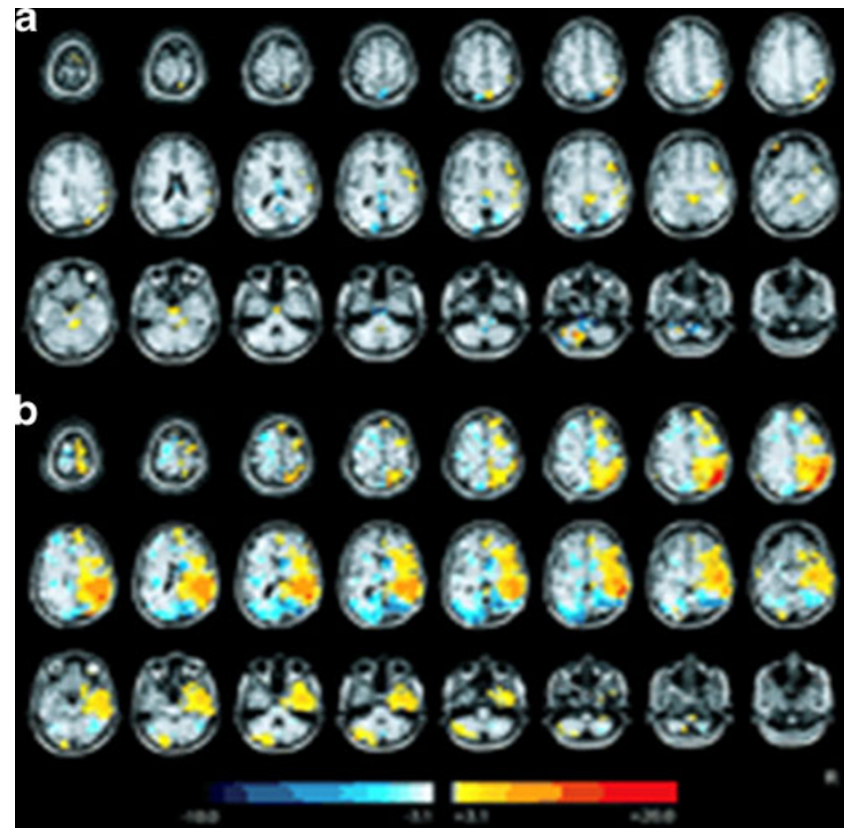

Fig. 1 fMRI imaging of the onset the micturition reflex a: normal patient, b: dispraxic patient.

Conclusion: In some dispraxic patient but not all, behavioral and pharmacological therapy appear to have an effect on the formation of mirror neuronal circuits, reasonably for existence of different level and typology of disease.

These findings suggest that activity within the mirror neuron system is severely impaired in patients with dyspraxia and enuresis-encopresis and provide a neural basis for therapeutical interventions and novel rehabilitation treatments. The fMRI can be useful for diagnosis and prediction of developmental dyspraxia because there is the possibility of a differentiation between type of dispraxia and prognosis.

A personalized approach to diagnosis and treatment of this patients should include a urological anamnesis with a neuro-urological approach and could help in the prevention of severe psychological distress and complication.

\section{PERSONALIZATION OF MULTIPLE SCLEROSIS TREATMENTS: THE CONCEPT OF CHELATION THERAPY APPROACH}

Zanella $S G^{1}$, Roberti di Sarcina $P^{2}$

${ }^{1}$ Expert in Chelation Therapy and Nutraceutics, Via Solferino, 45, 40124 Bologna, Italy

${ }^{2}$ High Council of Health, Ministry of Health, Italy Observatory and Methods for Health, Department of Sociology and Social Research, University of Milan-Bicocca, Italy

Multiple Sclerosis (MS) is one of the most common chronic diseases affecting the CNS with 2.5 million cases world- 
wide. The highest incidence in white women; three-quarters of cases present between ages 15 and 45. Symptoms are: fatigue, pain, visual impairment, imbalance, cognitive change, numbness, depression, spasticity, weakness, change in coordination, dysphagia, dizziness. Symptoms can be mild or severe, ongoing or intermittent, disabling or tolerable. It is characterized by acute multifocal demyelination. Environmental factors triggering chronic inflammatory response are unknown. The concomitant neuronal damage may be mediated by free radicals.

The traditional therapy: high-dose glucocorticoids, Glatiramer acetate (Copaxone), high-dose IFN beta 1 a (Rebif), high((Betaseron; Extavia) and low-dose IFN beta $1 \mathrm{~b}$ (Avonex), Natalizumab (Tysabri) and Mitoxandrone (Novantrone). All of these treatments have varying side effects and potential toxicity. Our hypothesis is that Aluminium toxicity is involved in the pathogenesis of MS. Aluminium is a common metal in the earth but not known to perform any function in the body: it is thought to be a cellular toxin and can damage the CNS. It is a potent environmental neurotoxin that induces locomotor, cognitive and behavioral disability. The best method of measuring the presence of toxic metals is EDTA Chelation Therapy.

Chelation Therapy consists of intravenous administration of a synthetic amino acid, ethylene diamine tetracetic acid (EDTA), given in combination with other substances. EDTA has been found to be effective in removing toxic metals from the body and EDTA chelation therapy was approved for this use by the Food and Drug Administration in 1953. At physiological pH EDTA removes toxic metals deposited in the body (body burden).

The fact is that some foods are health-promoting, others are health-depleting. Personalized treatment protocols base on a combination of chelation therapy for removing toxic metals, integration of vitamins, minerals and supplements to improve detoxification, essential fatty acids for antiinflammatory activity and diet correction by personalized programs. Glutathione (GSH) is a tripeptide molecule and plays a central role in cellular function, detoxification and protection from damage caused by free radicals. It is produced and circulates naturally in the body, neutralizing free radicals and removing environmental poisons. Deficiency of it contributes to oxidative stress and plays a key role in the pathogenesis of many diseases. Long chain Omega 3 fatty acids (EPA and DHA) are molecules that cannot be synthesized by humans. EPA and DHA reduce the levels of pro-inflammatory eicosanoids and promote wellness. Common sources include fish oil and some plant oils such as flax-seed. Reduction of high glycemic pro-inflammatory foods and regular physical activity are very important for the success by our protocol. Regular exercises increase the secretion of growth hormone (GH), stimulate the release of endorphins and decrease the risk of osteoporosis. 